\title{
Overexpression of microRNA-30a contributes to the development of aortic dissection by targeting lysyl oxidase
}

Yang Yu, MD, PhD, ${ }^{a}$ Enyi Shi, MD, PhD, ${ }^{\text {a }}$ Tianxiang Gu, MD, PhD, ${ }^{\text {a }}$ Rui Tang, $\mathrm{MD},{ }^{\text {a }}$ Shilun Gao, MD, Yongchao Wang, $\mathrm{MD}, \mathrm{PhD},{ }^{\mathrm{a}}$ and Hongbo Liu, $\mathrm{MD}, \mathrm{PhD}^{\mathrm{b}}$

\section{ABSTRACT}

Objective: To explore the role of microRNA (miR)-30a in the development of aortic dissection.

Methods: Human aortic specimens of aortic dissections and aneurysms were harvested. Aortic specimens from donors for heart transplantation served as controls. Rat aortic vascular smooth muscle cells (VSMCs) were transfected with agomiR-30a or antagomiR-30a, and control cells were incubated with empty vectors. Rats were pretreated with agomiR-30a or antagomiR-30a $\left(5 \times 10^{7}\right.$ transfection units every 3 days for 4 weeks), and empty vectors were infused to controls. Acute aortic dissection was induced by subcutaneous infusion of angiotensin II (1 $\mu \mathrm{g} \cdot \mathrm{kg}^{-1} \cdot \min ^{-1}$ for 24 hours). Protein expressions of lysyl oxidase (LOX) and elastin and gene expression of miR-30a were measured in VSMCs and human and rat aortic specimens by Western blot analysis and quantitative real-time polymerase chain reaction.

Results: Gene expression of miR-30a was much higher, and protein abundance of LOX and elastin was significantly lower, in the aortic dissection specimens $(P<.05$ vs controls). Transfection of agomiR-30a markedly decreased the luciferase activity of LOX in VSMCs of wild type, but not of LOX 3'-UTR mutant $(P=.002)$. In cultured VSMCs, transfection of agomiR-30a dramatically enhanced the gene expression of miR-30a and down-regulated the protein abundance of LOX and elastin $(P<.05$ vs controls). Pretreatment with agomiR-30a in vivo enhanced miR-30a expression and down-regulated the protein abundance of LOX and elastin in rat aortas $(P<.05$ vs controls). The rate of dissection was significantly higher in rats pretreated with agomiR-30a $(P=.003$ vs controls).

Conclusions: Overexpression of miR-30a contributes to the development of aortic dissection, possibly by targeting LOX. (J Thorac Cardiovasc Surg 2017;154:1862-9)

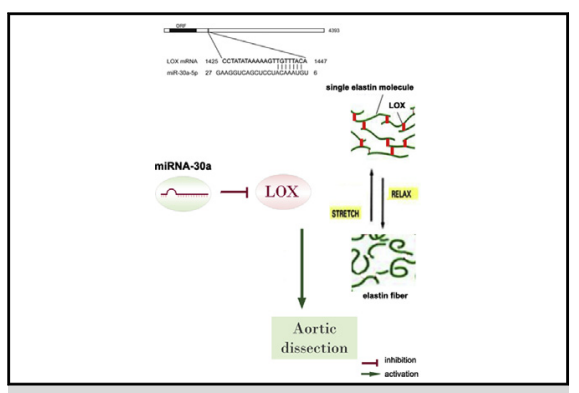

Proposed mechanism of miR-30a contributing to aortic dissections

\section{Central Message}

Overexpression of miR-30a contributes to the development of aortic dissection, possibly by inhibition of the LOX translation.

\section{Perspective}

The present study shows that miR-30a was overexpressed with a down-regulation of LOX in the aortic wall of rats with acute aortic dissection. Overexpression of miR-30a made the rat aortas more susceptible to Ang IIinduced dissection, possibly by inhibition of the protein translation of LOX. The current study provides novel insights into the molecular mechanism of aortic dissection and may open a door to preventive strategy for aortic dissection by targeting miRs.

See Editorial Commentary page 1870.
Acute aortic dissection, a major and fatal pathology of acute aortic syndrome, is initiated by an intimal tear and propagates within the medial layer of the aorta. Acute

From the ${ }^{\mathrm{a} D e p a r t m e n t}$ of Cardiac Surgery, First Affiliated Hospital; and ${ }^{\mathrm{b}}$ Department of Health Statistics, School of Public Health, China Medical University, Shenyang, China.

This work was supported by the National Natural Science Foundation of China (Grant 81471267).

Received for publication Nov 16, 2016; revisions received May 26, 2017; accepted for publication June 7, 2017; available ahead of print July 12, 2017.

Address for reprints: Tianxiang Gu, MD, PhD, Department of Cardiac Surgery, First Affiliated Hospital, China Medical University, Shenyang 110001, China (E-mail: gtxcmu@sina.com).

$0022-5223 / \$ 36.00$

Copyright (c) 2017 by The American Association for Thoracic Surgery

http://dx.doi.org/10.1016/j.jtcvs.2017.06.019 type A dissection has a mortality rate of $1 \%$ to $2 \%$ per hour during the first 24 to 48 hours after the onset of symptoms. ${ }^{1}$ Poorly controlled hypertension and mutations in genes for extracellular matrix constituents, membrane receptors, contractile proteins, and associated signaling molecules may be the main factors in aortic dissection. ${ }^{2}$

MicroRNAs (miRs) are important posttranscriptinal regulators. Previous studies have addressed the involvement

- Scanning this QR code will take you to a supplemental video for the article

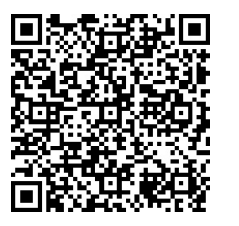




\section{Abbreviations and Acronyms \\ Ang = angiotensin \\ LOX = lysyl oxidase \\ $\mathrm{miR}=$ microRNA \\ qRT-PCR $=$ quantitative real-time polymerase chain reaction \\ VSMC $=$ vascular smooth muscle cell}

of miRs, including miR-195, miR-29b, miR-143, and miR-145, in aneurysm formation and complications. ${ }^{3-8}$ In comparison, the role of miRs in the formation of aortic dissection is still poorly understood.

Lysyl oxidase (LOX) and its related gene family members are a group of copper-dependent oxidodeaminases that cross-link lysyl residues on the structural proteins in the process of forming proper elastic lamellae and collagen fibers. ${ }^{9}$ LOX has an essential role in the development and function of the cardiovascular system. Inactivation of LOX is related to the development of aortic aneurysm and dissection. ${ }^{10,11}$ The TargetScan bioinformatics-based database (http://www.targetscan.org) identifies miR-30a as an endogenous regulator of LOX. ${ }^{12}$ LOX has been identified as the targeted protein of miR-30a in anaplastic thyroid cancer. ${ }^{13}$

The present study was conducted to measure the gene expression of miR-30a and the protein abundance of LOX in patients with acute aortic dissection, and to explore the possible role of miR-30a in the development of aortic dissection as an endogenous regulator of LOX in cultured rat aortic VSMCs and a rat model of acute aortic dissection.

\section{MATERIALS AND METHODS Clinical Cohorts}

Specimens of ascending aorta of patients with acute aortic dissections ( $\mathrm{n}=21)$ and ascending aortic aneurysms $(\mathrm{n}=18)$ were obtained during surgical operations. Aortic specimens from donors for heart transplantation $(\mathrm{n}=5)$ served as normal controls. Tissue samples were kept in liquid nitrogen for Western blot analysis and quantitative real-time polymerase chain reaction (qRT-PCR). The paraffin-embedded specimen was used for histological examination. This study was approved by the Ethics Review Committee of China Medical University (Shenyang, China), and all patients provided written informed consent.

\section{Lentivirus Vectors}

Lentivirus vector was used to transfer agomiR and antagomiR in vitro and in vivo. ${ }^{14,15}$ Chemically modified agomiR-30a and antagomiR-30a lentivirus gene transfer vectors were constructed by Genechem (Shanghai, China). Empty lentivirus agomiR-vectors or antagomiR-vectors were used as controls. All lentivirus vectors were prepared and titered to $1 \times 10^{9}$ transfection units $\cdot \mathrm{mL}^{-1}$.

\section{Luciferase Assays}

VSMCs were seeded in triplicate into a 96-well plate and cultured for 24 hours. VSMCs of LOX $3^{\prime}$-UTR wild type or LOX $3^{\prime}$-UTR mutant type were transfected with lentivirus vectors with or without
agomiR-30a, respectively. Luciferase activity was measured at 24 hours after transfection using the Light Switch Luciferase Assay System (Switchgear Genomics, Carlsbad, Calif), following the manufacturer's instructions.

\section{Cell Culture and Transfection In Vivo}

The rat aortic VSMC cell line was purchased from the cell bank of the Chinese Academy of Sciences, Beijing and cultured in vitro following a previously published protocol previously. ${ }^{16}$ The cultured VSMCs from the third passage to the fifth passage were infected with agomiR-30a, agomiR-vector, antagomiR-30a, or antagomiR-vector suspension by incubation for 1 hour at a multiplicity of infection of 10 in sextuplicate. The control VSMCs were cultured with normal medium without any vectors. After infection, VSMCs were continuously cultured with normal culture medium for the next 24 hours, after which VSMCs were harvested for analysis of gene expression of miR-30a and protein expression of LOX and elastin.

\section{Rat Model of Aortic Dissection}

Male Sprague-Dawley rats weighing approximately $350 \mathrm{~g}$ were used in all experiments. The experimental protocol was approved by the Ethics Review Committee of China Medical University, Shenyang. Acute aortic dissection was induced by continuous subcutaneous injection of angiotension (Ang) II $\left(1 \mu \mathrm{g} \cdot \mathrm{kg}^{-1} \cdot \mathrm{min}^{-1}\right)$ for 24 hours, followed by aortography. Then animals were then sacrificed for autopsy. Tissue samples of ascending aorta were kept in liquid nitrogen for Western blot analysis and qRT-PCR. The paraffin-embedded specimen was used for histological examination. Rats were divided at random into 5 groups, as shown in Figure 1. The control group $(\mathrm{n}=14)$ received only infusion of Ang II after feeding for 4 weeks. In the other 4 groups, agomiR-vector $(n=15)$, agomiR-30a $(\mathrm{n}=16)$, antagomiR-vector $(\mathrm{n}=15)$, or antagomiR-30a $(\mathrm{n}=15)$ was administered by intravenous injection $\left(5 \times 10^{7}\right.$ transfection units, every 3 days) for 4 weeks before infusion of Ang II.

\section{Histological Examination}

Paraffin-embedded sections ( $4 \mu \mathrm{m})$ of aortic tissues of human and rat were stained with hematoxylin and eosin and Verhoeff's Van Gieson stain.

\section{Western Blot Analysis}

LOX and elastin in aortic tissue (human and rat) and VSMCs were analyzed by sodium dodecyl sulfate-polyacrylamide gel electrophoresis and immunoblotted with anti-LOX or anti-elastin antibodies (Abcam, Cambridge, Mass). The optical density for each band on the Western blot was quantified using ImageJ (National Institutes of Health, Bethesda, $\mathrm{Md})$ and quantified as a relative fold to the control after normalization with $\beta$-actin.

\section{miR Extraction and qRT-PCR}

Total RNA of cultured VSMCs and aortic tissues was isolated using Trizol reagent (Invitrogen, Carlsbad, Calif), according to the manufacturer's instructions. The first-strand complementary DNA was generated using the Reverse-Transcription System Kit (Invitrogen). qRT-PCR was performed following a standard protocol using the StepOne Plus system (Applied Biosystems, Foster City, Calif). The U6 served as an internal control. Changes in expression were determined by the $2^{-\Delta \Delta \mathrm{CT}}$ method and expressed as relative fold to the control.

\section{Statistical Analysis}

Parametric values (protein expression of LOX and elastin, gene expression of miR-30a) are reported as mean \pm standard deviation. Differences were analyzed by 1-way analysis of variance followed by Bonferroni correction for post hoc testing. The incidence of aortic 


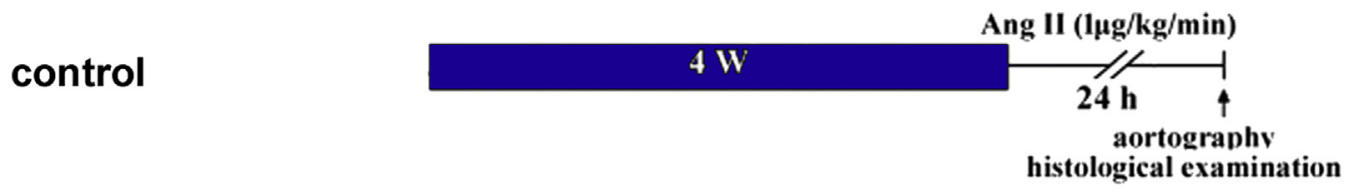

agomiR-vector

agomiR-30a

antagomiR-vector

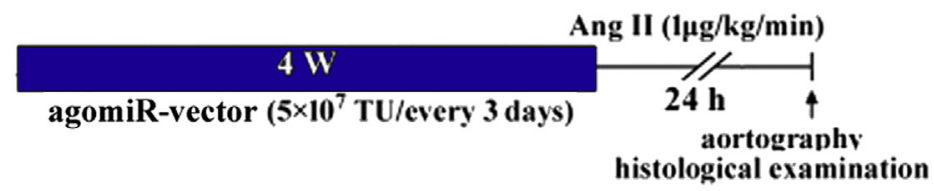

histological examination

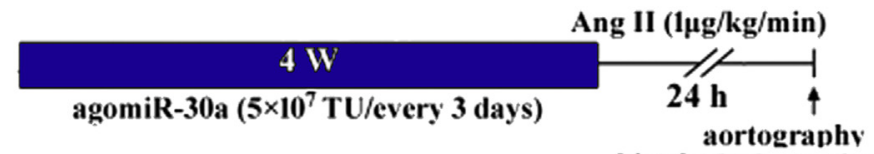

histological examination
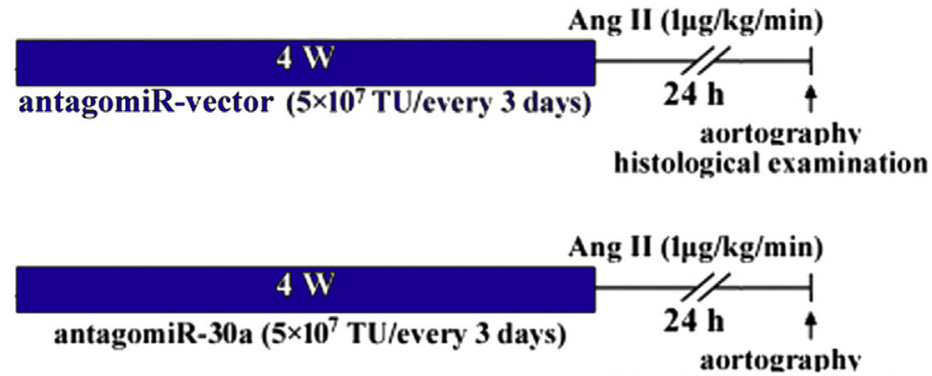

histological examination

FIGURE 1. Experimental groups and protocol. Ang II, Angiotensin II; miR-30a, microRNA-30a.

dissection in rat model was analyzed by a multiple-group $\chi^{2}$ test with Bonferroni correction $(P<.005$ before Bonferroni correction was considered statistically significant). Statistical significance was defined as $P<.05$. Statistical analysis was performed using SPSS version 19.0 (IBM, Armonk, NY).

\section{RESULTS}

\section{Histological Examination}

Hematoxylin and eosin-stained sections of aortic dissection showed rupture of media and extracellular matrix arranged loosely and in random, quite different from that of normal aorta and aneurysm. EVG staining revealed the elastin fibers in aortic specimens. Disorder and rupture of the elastin fibers were also pathological features of aortic dissection.

\section{Gene Expression of miR-30a and Protein Expression of LOX and Elastin in Aortic Specimens of Patients With Aortic Dissection}

Gene expression of miR-30a was significantly higher in aortic dissection specimens than in normal aorta $(P=.036)$ (Figure 2, $A)$. It was also higher in aortic aneurysm specimens compared with normal aorta, but the difference did not reach statistical significance $(P=.054)$ (Figure 2, A).
The protein abundance of LOX and elastin was significantly decreased in aortic dissection specimens compared with normal aorta $(P=.027$ and .031, respectively) (Figure 2, $B$ and $C$ ). There was no significant difference in protein expression of LOX and elastin between normal aorta and aneurysm specimens $(P=.062$ and .071, respectively) (Figure 2, $B$ and $C$ ).

\section{Luciferase Assays}

miR-30a significantly inhibited the luciferase activity of VSMCs of LOX $3^{\prime}$-UTR wild type $(P=.002)$ (Figure 3$)$. In contrast, such an inhibitory effect was essentially abolished when the putative miR-30a target sequence was deleted from the VSMCs of mutant LOX $3^{\prime}$-UTR $(P=.902)$.

\section{Gene Expression of miR-30a and Protein Expression of LOX and Elastin in VSMCs}

In the cultured VSMCs, agomiR-30a significantly increased the gene expression of miR-30a $(P=.003$ vs control) (Figure 4, A), whereas antagomiR-30a did not decrease the gene expression of miR-30a to a statistically significant extent ( $P=.07$ vs control) (Figure 4, $A$ ). agomiR-30a also significantly decreased protein abundance of LOX and elastin in the cultured VSMCs compared with 


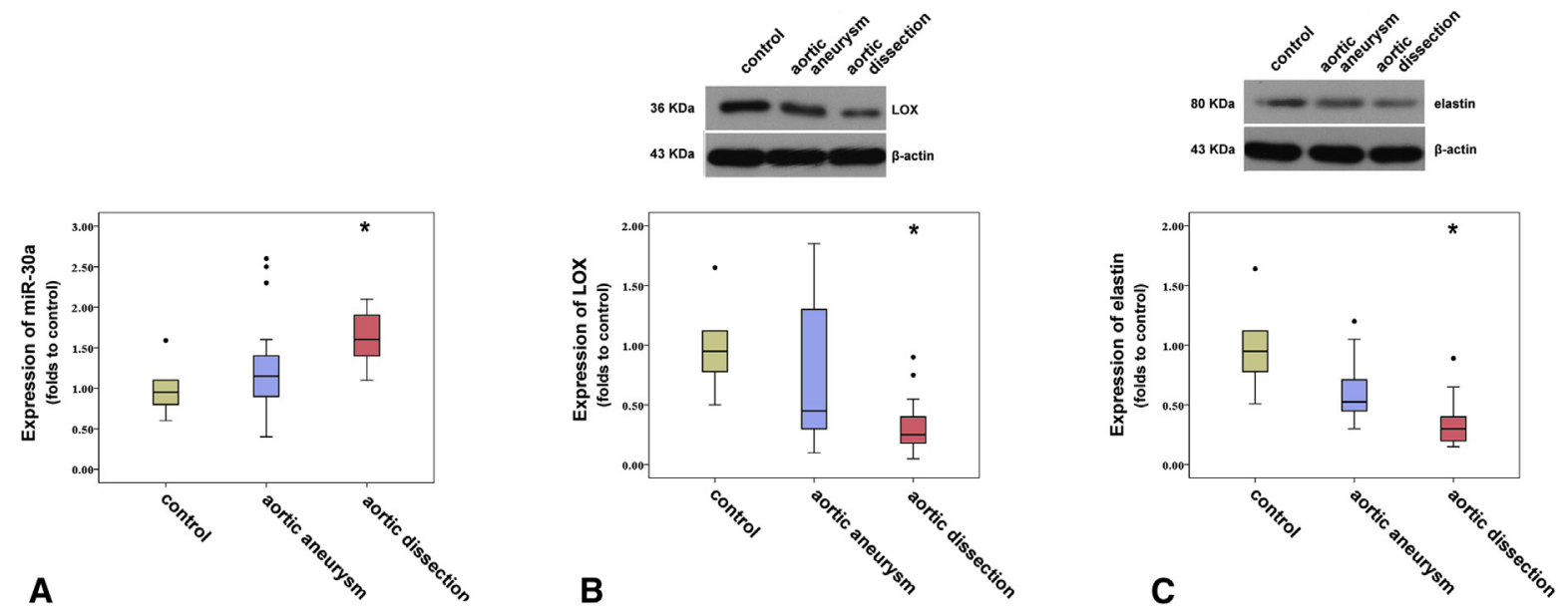

FIGURE 2. Gene expression of miR-30a and protein expression of LOX and elastin in specimens of normal ascending aorta $(\mathrm{n}=5)$, ascending aorta aneurysm $(\mathrm{n}=18)$, and acute aortic dissection $(\mathrm{n}=21)$. A, Densitometric quantification of miR-30a gene expression. B, Representative Western blot showing LOX protein expression and densitometric quantification of LOX gene expression. C, Representative Western blot showing elastin protein expression and densitometric quantification of elastin protein expression. In the box-and-whiskers graphs, the middle horizontal line represents the median, the upper and lower whiskers represent the maximum and minimum values of nonoutliers, and the extra dots represent outliers. $* P<.05$ versus control. miR-30a, MicroRNA-30a; LOX, lysyl oxidase.

the controls $(P=.012$ and .025 , respectively, vs control) (Figure $4, B$ and $C$ ).

\section{Gene Expression of miR-30a and Protein Expressions of LOX and Elastin in the Rat Model}

Transfection of agomiR-30a dramatically enhanced the gene expression of miR-30a $(P=.009$ vs control $)$

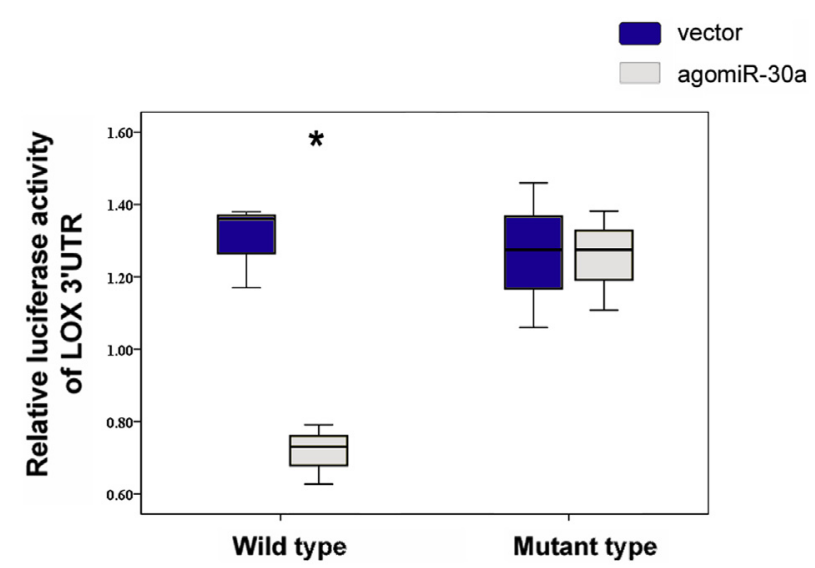

FIGURE 3. Luciferase activity in the presence of the agomiR-30a. miR-30a significantly inhibited the luciferase activity of VSMCs of LOX 3'-UTR wild type, whereas such an inhibitory effect was essentially abolished when the putative miR-30a target sequence was deleted from the VSMCs of mutant LOX $3^{\prime}$-UTR. The data indicate that miR-30a decreases the protein expression of LOX by directly interacting with the $3^{\prime}$ UTR of its mRNA. In the box-and-whiskers graphs, the middle horizontal line represents the median, the upper and lower whiskers represent the maximum and minimum values of nonoutliers, and the extra dots represent outliers. ${ }^{*} P=.002$ versus vector. $m i R-30 a$, MicroRNA-30a; LOX, lysyl oxidase.
(Figure 5, A) and inhibited protein abundance of LOX and elastin in rat aortas $(P=.011$ and .009 , respectively, vs control) (Figure 5, A). Transfection of antagomiR-30a did not significantly decrease the gene expression of miR-30a in rat aortas $(P=.071$ vs control) (Figure 5, A). No significant difference in protein expression of LOX was detected between the control rats and rats pretreated with antagomiR-30a $(P=.062)$ (Figure 5, B). Pretreatment of antagomiR-30a significantly enhanced the protein expression of elastin in rat aortic wall $(P=.007)$ (Figure 5, C).

\section{Incidence of Aortic Dissection in the Rat Model}

One rat in the agomiR-30a group died approximately 16 hours after Ang II-injection, due to rupture of aortic dissection as identified at autopsy. Figure 1, $A$, presents a representative aortogram for this rat showing the acute aortic dissection. The aortic dissection was further confirmed by autopsy (Figure 6, A). Immunohistochemical staining showed a reduction of LOX protein in the media of the aortic wall (Figure 6, A). Overexpression of miR-30a in rats significantly enhanced the incidence of aortic dissection induced by infusion of Ang II ( $P=.003$ vs the control group) (Figure 6, $B$ ).

\section{DISCUSSION}

The salient findings of the present study can be summarized as follows. miR-30a was overexpressed in the aortic wall of patients with acute aortic dissection, whereas the protein abundance of LOX was downregulated. As the target of miR-30a, protein abundance of LOX was negatively regulated by miR-30a in cultured rat 

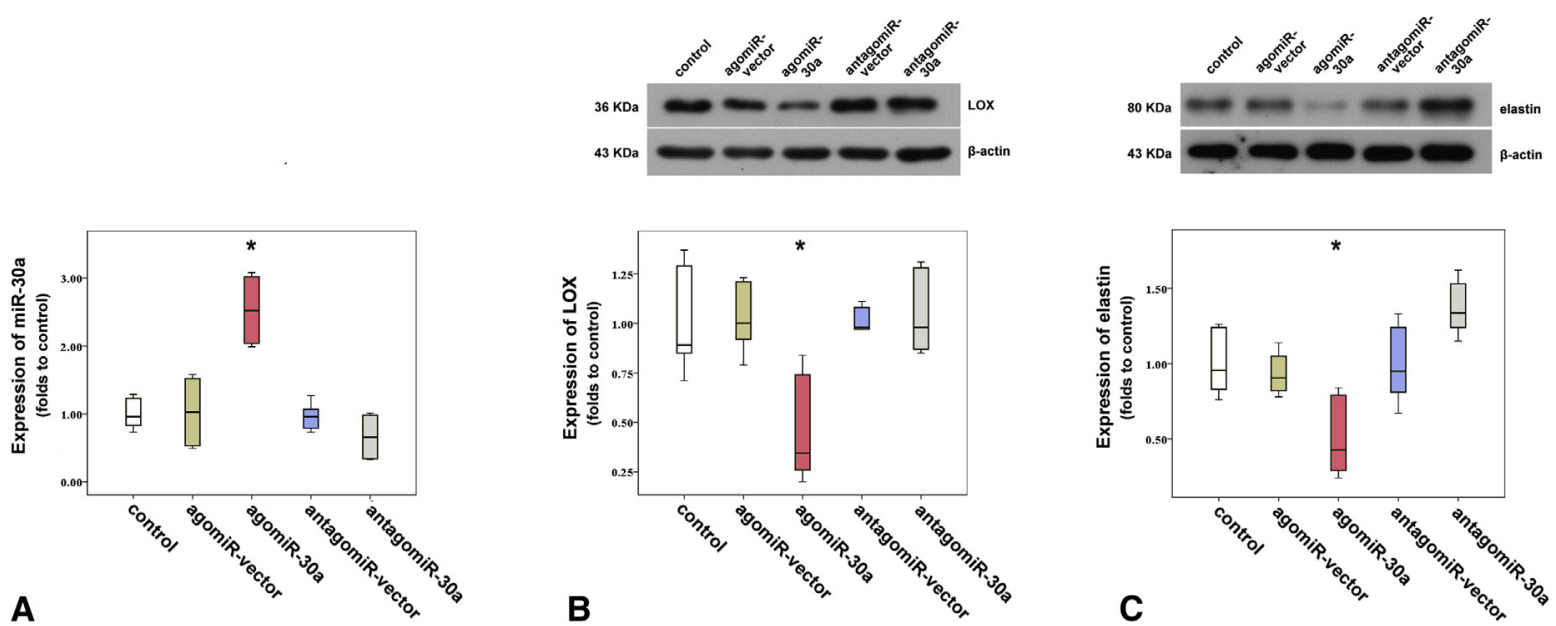

FIGURE 4. Expression of miR-30a, LOX, and elastin in rat VSMCs transfected with agomiR-30a or antagomiR-30a. A, miR-30a gene expression. B, Representative Western blot showing LOX expression and densitometric quantification of LOX protein expression. C, Representative Western blot showing elastin protein expression and densitometric quantification of elastin protein expression. In the box-and-whiskers graphs, the middle horizontal line represents the median, the upper and lower whiskers represent the maximum and minimum values of nonoutliers, and extra dots represent outliers. $* P<.05$ versus control. miR-30a, MicroRNA-30a; LOX, lysyl oxidase.

VSMCs. Overexpression of miR-30a made the rat aortas more susceptible to Ang II-induced dissection, possibly by inhibition of the protein translation of LOX.

As a posttranscriptional regulator of protein translation, the predominant role of miRs in the aortic pathologies has been illuminated through several studies. miRs have emerged as major intracellular players in pathophysiological mechanisms leading to aneurysm formation involving inflammatory processes, degradation of the extracellular matrix, and loss of smooth muscle cells. ${ }^{17}$ miR-145 has been confirmed as a novel VSMC phenotypic marker and modulator that controls vascular neointimal lesion formation. ${ }^{18}$ The gene expression of miR-143 and miR-145 was found to be significantly lower in human aortic aneurysms compared with control aortas. ${ }^{8}$ miR-29mediated down-regulation of extracellular matrix proteins has been shown to sensitize the aorta to the formation of aneurysms in advanced age. ${ }^{5}$ In 2 mouse aneurysm models, increased gene expression of miR-29b and decreased collagen gene expression were shown to augment aneurysm growth. ${ }^{4}$ Overexpression of miR-29b has been suggested to be important to the pathogenesis of
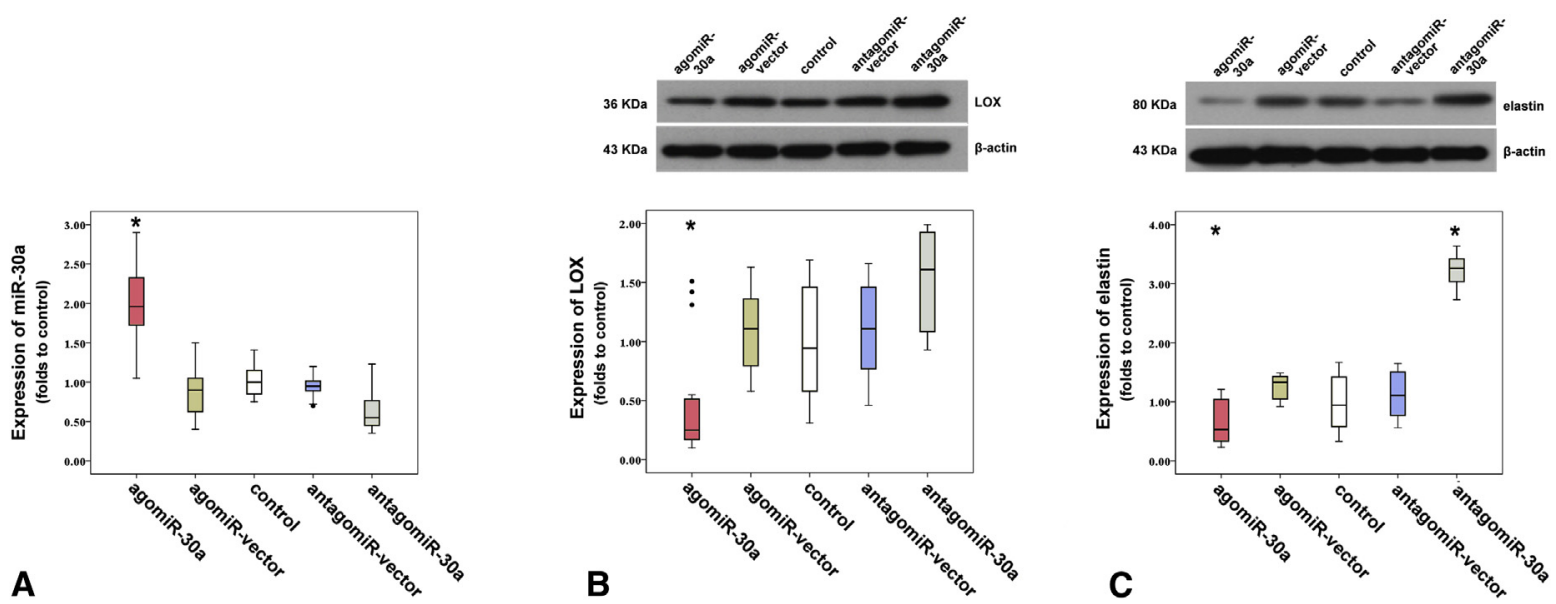

FIGURE 5. Gene expression of miR-30a and protein expression of LOX and elastin in rats pretreated with agomiR-30a or antagomiR-30a (control group, $\mathrm{n}=14$; agomiR-vector group, $\mathrm{n}=15$; agomiR-30a group, $\mathrm{n}=16$; antagomiR-vector group, $\mathrm{n}=15$; antagomiR-30a group, $\mathrm{n}=15$ ). A, miR-30a gene expression. B, Representative Western blot showing LOX protein expression and densitometric quantification of LOX protein expression. C, Representative Western blot showing elastin protein expression and densitometric quantification of elastin protein expression. The upper and lower borders of the box represent the upper and lower quartiles. In the box-and-whiskers graphs, the middle horizontal line represents the median, the upper and lower whiskers represent the maximum and minimum values of nonoutliers, and the extra dots represent outliers. $* P<.05$ versus control. miR-30a, MicroRNA-30a; $L O X$, lysyl oxidase. 

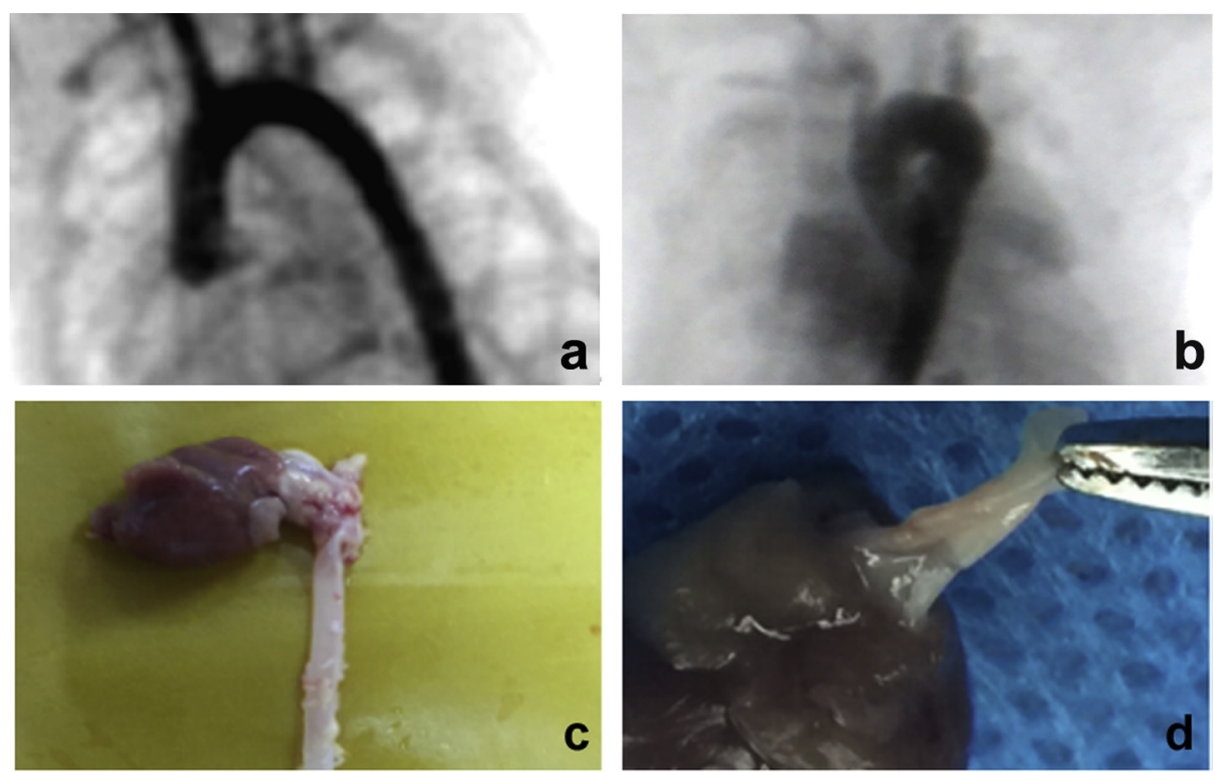

A
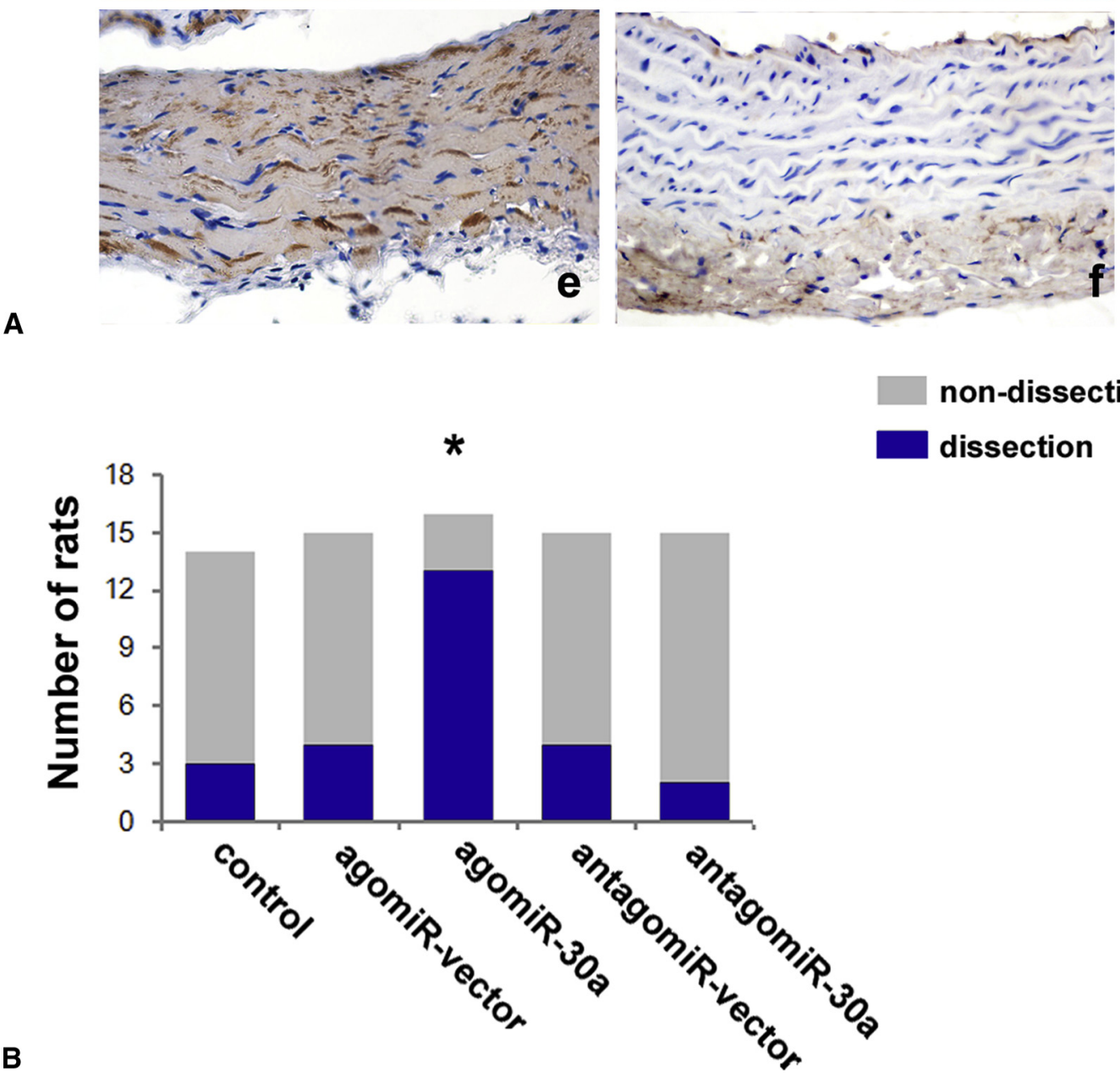

non-dissection

dissection

FIGURE 6. A, Aortography (a and b), autopsy ( $\mathrm{c}$ and d), and immunohistochemical staining pictures for LOX (e and f) of normal rat (a, c, and e) and rat with aortic dissection $(\mathrm{b}, \mathrm{d}$, and $\mathrm{f}$ ). $\mathrm{B}$, Incidence of aortic dissection in the rat model. $* P=.003$ versus control. miR-30a, MicroRNA-30a. 


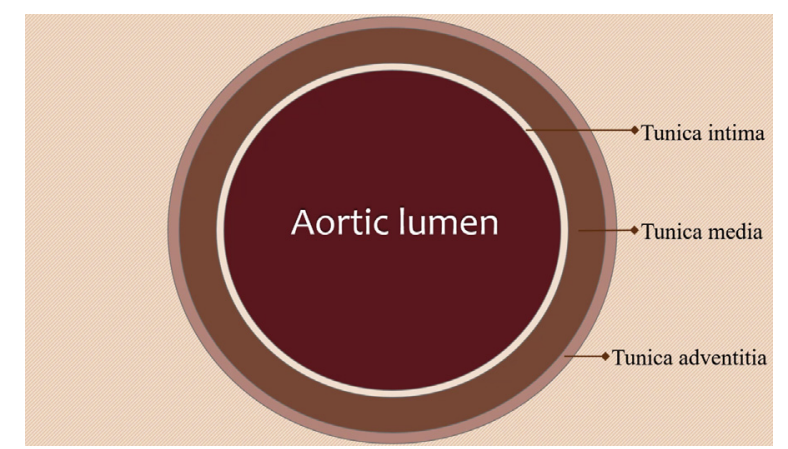

VIDEO 1. The proposed mechanism of miR-30a contributing to aortic dissections. LOX is an enzyme responsible for the cross-linking of collagen and elastin, the major structural components of the arterial wall. LOX is a predicted to be a target protein of miR-30a by a bioinformatics-based database. Overexpression of miR-30 inhibits the protein expression of LOX. Loss of LOX would weaken the aortic wall and make the aorta prone to dissection. Video available at: http://www.jtcvsonline.org/article/S00225223(17)31229-1/fulltext.

early aneurysm development in Marfan syndrome, and miR-29b blockade was found to prevent early aneurysm development by regulating aortic wall apoptosis and extracellular matrix abnormalities. ${ }^{6}$ miR-195 represents a potent regulator of the aortic extracellular matrix and contributes to the pathogenesis of aortic aneurysmal disease. miR-195 also might serve as a noninvasive biomarker of abdominal aortic aneurysms. ${ }^{3}$

Aortic aneurysm and aortic dissection are 2 separate but closely related entities. Compared with the extensive studies done in aortic aneurysms, research on the role of miRs in the development of aortic dissection remains limited. Using microarray techniques, a set of differentially expressed miRs (18 up-regulated and 56 down-regulated) was revealed in tissue of aortic dissections compared to normal aortas. The miR-29 and miR-30 families likely play roles in the regulation of focal adhesion and the mitogen-activated protein kinase signaling pathways. ${ }^{19}$ Down-regulation of miR-29 significantly increased the protein expression of aortic collagen types I and III in patients with aortic dissection, which likely was responsible for the aortic distensibility and systemic compliance. ${ }^{20}$

In the present study, the role of miR-30a in aortic dissection was highlighted. In human aortic tissue specimens, the gene expression of miR-30a was significantly higher in patients with aortic dissection compared with patients with normal aortas. Furthermore, up-regulation of the gene expression of miR-30a by lentivirus vectors containing agomiR-30a promoted the development of Ang II-induced aortic dissections in the rat model. miR-30a has been shown to be expressed in the cerebral vessels of rats ${ }^{21}$ and stimulate arteriolar branching. ${ }^{22}$ Our results provide the first evidence that overexpression of miR-30a contributes to the pathogenesis of aortic dissections.
Several proteins have been suggested as targets of miR-30a in different tissues, such as snail $1,{ }^{23}$ glucoseregulated protein, ${ }^{24}$ and Notch ligand Dll4. ${ }^{22}$ LOX is also a predicated target of miR-30a according to the TargetScan bioinformatics-based database (http://www.targetscan.org). In the present study, the luciferase assays of the cultured rat VSMCs indicate that miR-30a is bound to the $3^{\prime}$-UTR of rat LOX mRNA and negatively regulates LOX protein expression. Transfection of agomiR-30a in vivo significantly down-regulated the protein abundance of LOX in the rat aorta. These data confirm that LOX is also a direct target of miR-30a.

LOX is an enzyme responsible for the cross-linking of collagen and elastin, the major structural components of the arterial wall, both in vitro and in vivo. ${ }^{9}$ Mutant LOX null mice exhibited highly abnormal aortic histology characterized by fragmented elastic fibers and aberrant smooth muscle cell layers. ${ }^{10}$ LOX-targeted mice $(\operatorname{LOX}(-/-))$ died soon after parturition and showed cardiovascular instability with ruptured arterial aneurysms, indicating that LOX is critical during embryogenesis for structural stability of the aorta. ${ }^{25}$ Guo and colleagues reported that the deficiency of LOX in mice or inhibition of LOX in turkeys and rats caused aortic dissections. ${ }^{26}$ Clinical data suggest that a missense mutation in LOX is associated with aortic disease in humans, likely through insufficient cross-linking of elastin and collagen in the aortic wall. ${ }^{11}$ In the present study, protein levels of LOX and elastin in the specimens of aortic dissections were much lower than those in normal aortas. The incidence rate of acute Ang II-induced aortic dissection was significantly increased in rats transfected with agomiR-30a, in which protein abundance of LOX and elastin in the aorta was markedly down-regulated. Although the decreased protein abundance of elastin was found in combination with overexpression of miR-30a in human aortic specimens as well as in cultured rat VSMCs and rat aortic tissues, bioinformatics analysis did not suggest elastin as a target of miR-30a. LOX was shown to initiate the formation of covalent cross-linking of collagens and elastin. Administration of a LOX inhibitor for 8 weeks blocked collagen accumulation and aggravated elastic fiber impairment. ${ }^{27}$ Highly purified bovine aorta LOX peptide was found to increase matrix elastin synthesis in cultured VSMCs by $40 \%$ to $80 \%$ over that in control cultures in a dose-dependent manner. ${ }^{28}$ Therefore, elastin may be a downstream protein regulated by LOX. Collectively, findings indicate that overexpression of miR-30a contributes to the development of Ang II-induced aortic dissection in rats by targeting LOX directly. Down-regulation of protein abundance of LOX and elastin may be a major molecular mechanism for the pathological changes of the aortic wall in dissections. Video 1 summarizes the possible mechanism by 
which miR-30a contributes to the development of aortic dissections.

This study has several limitations that warrant comment. Other target proteins besides LOX that also may be implicated in the function of overexpression of miR-30a should be identified in detail. Moreover, gene expression of miR-30a in the aortic specimen does not reflect the clinical condition before the onset of aortic dissection. Finally, the rat model of Ang II-induced aortic dissection used in this study cannot fully mimic the development of aortic dissection in humans.

\section{CONCLUSIONS}

Taken together, our findings indicate that overexpression of miR-30a contributes to the development of aortic dissection, possibly through inhibition of the protein abundance of LOX in the aortic wall. This study provides novel insights into the molecular mechanisms of aortic dissection and may open a door to a preventive strategy for aortic dissection by targeting miRs.

\section{Conflict of Interest Statement}

Authors have nothing to disclose with regard to commercial support.

\section{References}

1. Hagan PG, Nienaber CA, Isselbacher EM, Bruckman D, Karavite DJ, Russman PL, et al. The International Registry of Acute Aortic Dissection (IRAD): new insights into an old disease. JAMA. 2000;283:897-903.

2. Humphrey JD, Schwartz MA, Tellides G, Milewicz DM. Role of mechanotransduction in vascular biology: focus on thoracic aortic aneurysms and dissections. Circ Res. 2015;116:1448-61.

3. Zampetaki A, Attia R, Mayr U, Gomes RS, Phinikaridou A, Yin X, et al. Role of miR-195 in aortic aneurysmal disease. Circ Res. 2014;115:857-66.

4. Maegdefessel L, Azuma J, Toh R, Merk DR, Deng A, Chin JT, et al. Inhibition of microRNA-29b reduces murine abdominal aortic aneurysm development. J Clin Invest. 2012;122:497-506.

5. Boon RA, Seeger T, Heydt S, Fischer A, Hergenreider E, Horrevoets AJ, et al. MicroRNA-29 in aortic dilation: implications for aneurysm formation. Circ Res. 2011;109:1115-9.

6. Merk DR, Chin JT, Dake BA, Maegdefessel L, Miller MO, Kimura N, et al. miR-29b participates in early aneurysm development in Marfan syndrome. Circ Res. 2012;110:312-24.

7. Cordes KR, Sheehy NT, White MP, Berry EC, Morton SU, Muth AN, et al. miR-145 and miR-143 regulate smooth muscle cell fate and plasticity. Nature. 2009;460:705-10.

8. Elia L, Quintavalle M, Zhang J, Contu R, Cossu L, Latronico MV, et al. The knockout of miR-143 and -145 alters smooth muscle cell maintenance and vascular homeostasis in mice: correlates with human disease. Cell Death Differ. 2009; 16:1590-8.

9. Smith-Mungo L, Kagan HM. Lysyl oxidase: properties, regulation and multiple functions in biology. Matrix Biol. 1998;16:387-98.

10. Mäki JM, Räsänen J, Tikkanen H, Sormunen R, Mäkikallio K, Kivirikko KI, et al. Inactivation of the lysyl oxidase gene Lox leads to aortic aneurysms, cardiovascular dysfunction, and perinatal death in mice. Circulation. 2002 106:2503-9.

11. Lee VS, Halabi CM, Hoffman EP, Carmichael N, Leshchiner I, Lian CG, et al Loss of function mutation in LOX causes thoracic aortic aneurysm and dissection in humans. Proc Natl Acad Sci U S A. 2016;113:8759-64.

12. Lewis BP, Shih IH, Jones-Rhoades MW, Bartel DP, Burge CB. Prediction of mammalian microRNA targets. Cell. 2003;115:787-98.

13. Boufraqech M, Nilubol N, Zhang L, Gara SK, Sadowski SM, Mehta A, et al miR30a inhibits LOX expression and anaplastic thyroid cancer progression. Cancer Res. 2015;75:367-77.

14. Wang Y, Gu T, Shi E, Yu L, Wang C, Zhang Y, et al. Inhibition of microRNA-29c protects the brain in a rat model of prolonged hypothermic circulatory arrest. J Thorac Cardiovasc Surg. 2015;150:675-84.

15. He F, Shi E, Yan L, Li J, Jiang X. Inhibition of micro-ribonucleic acid-320 attenuates neurologic injuries after spinal cord ischemia. $J$ Thorac Cardiovasc Surg. 2015;150:398-406.

16. Li J, Zhao L, He X, Yang T, Yang K. MiR-21 inhibits c-Ski signaling to promote the proliferation of rat vascular smooth muscle cells. Cell Signal. 2014;26:724-9.

17. Raffort J, Lareyre F, Clement M, Mallat Z. Micro-RNAs in abdominal aortic aneurysms: insights from animal models and relevance to human disease. Cardiovasc Res. 2016;110:165-77.

18. Cheng Y, Liu X, Yang J, Lin Y, Xu DZ, Lu Q, et al. MicroRNA-145, a novel smooth muscle cell phenotypic marker and modulator, controls vascular neointimal lesion formation. Circ Res. 2009;105:158-66.

19. Liao M, Zou S, Weng J, Hou L, Yang L, Zhao Z, et al. A microRNA profile comparison between thoracic aortic dissection and normal thoracic aorta indicates the potential role of microRNAs in contributing to thoracic aortic dissection pathogenesis. J Vasc Surg. 2011;53:1341-9.e3.

20. Wang X, LeMaire SA, Chen L, Shen YH, Gan Y, Bartsch H, et al. Increased collagen deposition and elevated expression of connective tissue growth factor in human thoracic aortic dissection. Circulation. 2006;114(1 Suppl): I200-5.

21. Müller AH, Povlsen GK, Bang-Berthelsen $\mathrm{CH}$, Kruse LS, Nielsen J, Warfvinge K, et al. Regulation of microRNAs miR-30a and miR-143 in cerebral vasculature after experimental subarachnoid hemorrhage in rats. BMC Genomics. 2015; $16: 119$.

22. Jiang Q, Lagos-Quintana M, Liu D, Shi Y, Helker C, Herzog W, et al. miR-30a regulates endothelial tip cell formation and arteriolar branching. Hypertension 2013:62:592-8.

23. Yuan CT, Li XX, Cheng QJ, Wang YH, Wang JH, Liu CL. MiR-30a regulates the atrial fibrillation-induced myocardial fibrosis by targeting snail 1. Int J Clin Exp Pathol. 2015;8:15527-36.

24. Chen M, Ma G, Yue Y, Wei Y, Li Q, Tong Z, et al. Downregulation of the miR-30 family microRNAs contributes to endoplasmic reticulum stress in cardiac muscle and vascular smooth muscle cells. Int J Cardiol. 2014;173:65-73.

25. Hornstra IK, Birge S, Starcher B, Bailey AJ, Mecham RP, Shapiro SD. Lysy oxidase is required for vascular and diaphragmatic development in mice. J Biol Chem. 2003;278:14387-93.

26. Guo DC, Regalado ES, Gong L, Duan X, Santos-Cortez RL, Arnaud P, et al. LOX mutations predispose to thoracic aortic aneurysms and dissections. Circ Res. 2016;118:928-34.

27. Busnadiego O, Gorbenko Del Blanco D, González-Santamaría J, Habashi JP Calderon JF, Sandoval P, et al. Elevated expression levels of lysyl oxidases protect against aortic aneurysm progression in Marfan syndrome. J Mol Cell Cardiol. 2015;85:48-57.

28. Kothapalli CR, Ramamurthi A. Lysyl oxidase enhances elastin synthesis and matrix formation by vascular smooth muscle cells. J Tissue Eng Regen Med. 2009;3:655-61.

Key Words: microRNA-30a, aortic dissection, lysyl oxidase 